\title{
Entre las bestias y los dioses: Naturaleza y artificio en Jacques Rancière y Leo Strauss
}

\author{
Octavio Majul ${ }^{1}$ \\ CONICET- IIGG- FSOC-UBA \\ omajulcg@gmail.com
}

Recibido 4/07/2018

Aprobado 9/08/2018

\section{Resumen:}

El presente artículo contrapondrá el modo en el que Jacques Rancière y Leo Strauss piensan la relación entre lo natural y lo artificial, entre physis y nomos. Se abordará esta discusión a la luz de la pregunta por la posibilidad de una teoría política que supere el momento de la negatividad. La restitución de los modos de relación entre physis y nomos que el autor francés permite pensar consistirá en la primera parte del trabajo. La segunda intentará ir más allá de los modos planteados por Rancière mediante una reposición del argumento de Leo Strauss sobre las enseñanzas de la filosofía política, en particular aquellas en torno a la relación entre lo natural y lo artificial.

Palabras Clave: Rancière - Leo Strauss - Política -Posfundacionalismo- Negatividad

\section{Abstract}

The present article compares how Jacques Rancière and Leo Strauss think the relation between the natural and the artificial, between physis and nomos. This discussion will be approached under the light of the question of the possibility of a political theory that surpass the negativity moment. The restitution of the ways in which the French author link physis and nomos will take part in the first section of the article. The second one will go further Rancière's ways through Leo Strauss's arguments of political

\footnotetext{
${ }^{1}$ Licenciado en Ciencia Política por la Facultad de Ciencias Sociales de la Universidad de Buenos Aires, becario doctoral del Consejo Nacional de Investigaciones Científicas y Técnicas con lugar de trabajo en el Instituto de Investigaciones Gino Germani, doctorando en Ciencias Sociales en la Universidad de Buenos Aires.
} 
philosophy's teachings, particularly those about the relation between the natural and the artificial.

Keywords: Rancière - Leo Strauss - Politics -Posfundacionalism- Negativity

El posfundacionalismo es un hecho. En mayor o menor medida las discusiones contemporáneas de la teoría y filosofía política deben vérselas con el pensamiento político posfundacional. O aún más, el lenguaje en el cual parte de la teoría y filosofía política viven está condicionado por la retórica posfundacional. Lenguaje que se trasluce en una economía conceptual determinada. Economía conceptual cuyos puntos álgidos se encuentran en conceptos tales como contingencia, apertura, heterogeneidad, incertidumbre, indeterminación, indecibilidad, conflicto, desacuerdo, etc. Con esto en mente, parecería existir un desfasaje entre el pensamiento político posfundacional y su economía conceptual. Es que si el posfundacionalismo no es la inversión del fundacionalismo -ya que el antifundacionalismo implicaría "un nuevo fundamento final, una suerte de 'antifundamento'"'2 - sino el reconocimiento de la necesidad y al mismo tiempo imposibilidad de todo fundamento -del juego interminable entre fundaciones y sus puestas en cuestión, positividad y negatividad, clausura y apertura- sus conceptos se encuentran de un solo lado de la doble lección: aquel delimitado por la apertura. Es el momento rupturista, de intensidad elevada, el único que permite aprehender lo indeterminado como tal y hacer justicia, en última instancia, a la incertidumbre de lo existente ${ }^{3}$

Es por ello, argumentamos, que el posfundacionalismo ha relegado el tratamiento de lo ordinario, el tratamiento de lo común. Más precisamente ha olvidado la indagación

${ }^{2}$ Marchart, Oliver. El pensamiento político posfundacional. Buenos Aires: FCE, 2009. 26.

${ }^{3}$ El desfasaje entre el pensamiento posfundacional y su retórica se podría explicar por el estatus privilegiado que ocupa la indagación de lo ontológico. Si en términos ontológicos la contingencia ocupa un lugar privilegiado, si la única certeza que existe es la imposibilidad de un fundamento último, el momento óntico de la apertura a la novedad, el de la mostración de lo existente como parcial parecería estar más la altura de lo ontológico que el momento de clausura. En esta línea toda fundamentación, todo orden, si bien lógicamente comprensible por la necesidad de la alternación entre el fundar y el desfundar, se ubica del lado del sí mismo. De ese lado que, en última instancia, es más acorde al principio esencialista del fundamento. 
de los modos de lo ordinario, de los modos de lo común. La acentuación del momento de la apertura conflictiva, de la puesta en cuestión del fundamento, ha imposibilitado el análisis de los diversos modos del fundamento. Anclado en el momento de la negatividad, el posfundacionalismo renuncia a pensar las formas de la positividad, el hecho de que no toda positividad tiene el mismo signo y que las variaciones de ésta son relevantes. Lo mismo sucede con la negatividad, ¿toda puesta en cuestión, toda manifestación del conflicto, es idéntica? ¿Es lo mismo oponerse a dos positividades de signo contrario?

Una época atenta a poner en tela de juicio las oposiciones binarias ha asistido a una proliferación de las mismas en el campo de la política: derecho-justicia, consensoconflicto, policía-política, instituciones-pueblo, etc. Mientras cada uno de los primeros conceptos de la oposición representa el momento de positividad que opera bajo el mecanismo de la identidad, del sí mismo; el segundo concepto viene a hacer saltar la repetición de lo uno al develar el carácter parcial del mismo. La acentuación en el carácter excepcional deja de lado la posibilidad de pensar las variaciones de lo común. En última instancia se relega el pensamiento sobre lo común, se renuncia a la articulación de un común. La simplificación de la oposición norma-excepción es contraproducente para pensar lo ordinario, es decir, el momento de vida por excelencia. Equivalente es lo que sucede con la identificación de la política con el conflicto. La política es un momento, siempre pasajero, de intensidad alta. Se opone al consenso y se destaca por la irrupción de un no ser en el seno de la comunidad. Esto obstaculiza pensar los modos de la política que incluyen relaciones no siempre coherentes de politización-despolitización. Obstaculiza pensar los modos del consenso.

En este sentido al presente artículo le subyace la motivación de pensar una política que supere el momento de la negatividad, es decir una política de la positividad. Una política que aún a sabiendas de que todo orden es parcial no extraiga como corolario la destrucción de todo orden. Está movida por la posibilidad de restituir un pensamiento sobre lo político atento a aquello que transcurre entre las dicotomías. Se preguntará, entonces, por la condición de posibilidad de un pensamiento tal. Eso nos llevará a discutir 
con dos pilares de la retórica posfundacional: el énfasis en lo excepcional y la identificación entre política y conflicto. Para esto se utilizará como caso paradigmático -lo cual determina, a su vez, el alcance de los resultados de la discusión- el pensamiento de Jacques Rancière, más precisamente, el modo en el cual piensa la relación entre lo natural y lo artificial, entre physis y nomos.

La restitución de los modos de relación entre physis y nomos que el autor francés permite pensar consistirá en la primera parte del trabajo. La segunda parte intentará ir más allá de los modos planteados por Rancière. Para ello una repondrá las enseñanzas que Leo Strauss obtiene de la filosofía política, en particular aquellas que giran en torno a la relación entre physis y nomos. La legitimidad de la utilización de Leo Strauss viene dada por su introducción vía Rancière. Es éste quien polemiza de modo directo con Strauss identificándolo con los intentos de un retorno a la arquipolítica platónica ${ }^{4}$. Esta identificación nos permitirá realizar una crítica los modos de relacionar physis y nomos planteados por Rancière a partir de una lectura diferente de la filosofía política de Leo Strauss.

\section{El espacio de la política o la naturaleza como lo múltiple proliferante}

Jacques Rancière es uno de los principales inscriptos en la tradición que sostiene los dos pilares de excepcionalismo e identificación entre política e intensidad. El modo en el cual articula ambos pilares es bajo la oposición entre orden policial y política definidos como dos modos de ser-juntos humanos. El orden policial establece una disposición de la comunidad, no importando el modo en el que aquella comunidad se disponga. La política suspende la disposición $\mathrm{X}$ actualizando la contingencia de la igualdad. Un modo no

${ }^{4}$ Tan sólo en El Desacuerdo Leo Strauss es mencionado implícita o explícitamente de manera continua. Es notoria su aparición en el inicio como en el cierre del mismo. Cfr. Rancière, Jacques. El desacuerdo. Política y filosofía. Buenos Aires: Nueva Visión, 2012. 5-12, 14-15, 31, 85-86, 119-120, 168173. Luciano Nosetto contrapone las lecturas que Rancière y Leo Strauss realizan de Platón y, en el mismo sentido que nosotros, afirma "Justifica el recurso a Strauss el hecho de que Rancière lo sindique como un exponente de los intentos de retorno al ideal republicano de Platón", en Nosetto, Luciano. "Jacques Rancière, Leo Strauss y el sentido del idealismo platónico”, Leviathan | Cuadernos de Investigación Política, N. 10, pp 59-79, 2015. 65. 
ortodoxo de analizar el problema se da a través de la lectura de la relación entre physis y nomos. En otras palabras, entre lo natural y lo artificial.

Tres son los modos que nos presenta Rancière de interrelación entre physis y nomos. El primero es el de la arquipolítica platónica y sus reactualizaciones contemporáneas $^{5}$. La arquipolítica pretende encontrar un principio de la comunidad, permitiéndole organizarse en torno a la distinción entre lo justo y lo injusto. La propuesta platónica sería la de fundar la ciudad en la encarnación de su principio, en la verdadera idea de bien, es decir, "poner la verdadera idea de la comunidad y del bien asociado a su naturaleza" 6 . En última instancia, el modelo de Platón "expone en toda su radicalidad el proyecto de una comunidad fundada sobre la realización integral, la sensibilización integral de la arkhé de la comunidad"7.

En la arquipolítica la comunidad realizada coincidiría con los bordes la comunidad posible. La anulación del conflicto, al realizar íntegramente la idea de justicia, es el horizonte de la arquipolítica. El intento platónico de fundar el nomos en la physis o, en otras palabras, de realizar la physis en le nomos, opera anulando el espacio entre ellos. La anulación de ese espacio supone la ausencia de todo vacío, I intento cerrar a la comunidad sobre sí misma. En palabras de Rancière: "La arquipolítica es la realización integral de la physis en nomos, el devenir sensible total de la ley comunitaria. No puede haber ni tiempo muerto ni espacio vacío en el tejido de la comunidad" ${ }^{8}$.

La arquipolítica, de este modo, aspira a clausurar el espacio político bajo el dominio de lo Uno, de la "comunidad que funciona en el régimen de lo Mismo" ${ }^{9}$. Pero dicho principio del sí mismo ${ }^{10}$, tanto como la equiparación entre physis y nomos, no

\footnotetext{
${ }^{5}$ La figura de la arquipolítica parece tener una prioridad mayor frente a los otros modos de la filosofía política tales como la metapolítica y la parapolítica. No sólo en términos cuantitativos, es la figura más citada, sino en términos cualitativos, es el elemento que elige Rancière para abrir y cerrar El desacuerdo.

${ }^{6}$ Rancière, Jacques. El desacuerdo. Óp. Cit., 2012. 86.

${ }^{7}$ Ibídem. 88.

${ }^{8}$ Ibídem. 91.

${ }^{9}$ Ibídem. 86.

${ }^{10}$ Ver Ibídem.87, 131, 136-7,151.
} 
corresponden solo a la arquipolítica. Rancière describe del mismo modo el orden policial y su manifestación por excelencia, él consenso:

Su principio consiste en establecer la convertibilidad permanente del Uno de la ley en el Uno del sentimiento que define el ser-juntos [...] El esquema de la ley consensual anuda así el orden del nomos como facultad de convenir y contratar con el orden de la physis como facultad de con-sentir. El consenso es una relación de circularidad entre la naturaleza y la ley, que deja a ésta la tarea de determinar la antinaturaleza que es experimentada por aquella como insoportable. La ley lo hace separando de la physis, concebida como potencia de lo que sale a la luz, a la antinaturaleza, o sea la potencia de lo múltiple proliferante ${ }^{11}$.

El esquema del orden policial es aquel del círculo, el régimen de lo Uno, del sí Mismo. Funciona al modo de la tautología. Esto lo obliga a identificar la naturaleza con su realización sensible expulsando, así, como antinatural, aquello que no puede ser subsumido por el sí mismo. La potencia de lo que sale a la luz -aquello que se muestra como natural- ocluye, de este modo, la potencia de lo múltiple proliferante. Esa oclusión es identificada por Rancière como un reparto de partes $X$ en una comunidad dada. En tanto todo reparto excluye incluyendo una parte sin parte, todo reparto mutila y reduce a la naturaleza a su realización sensible ${ }^{12}$.

En lo antedicho se vislumbra cual es la concepción de la physis en Rancière. La igualación entre physis y nomos implica la negación de la distorsión y, por tanto, de la política, ya que "es la distorsión convertida en imposible [...] la identidad entre política y policía" ${ }^{13}$. Por oposición, la distancia entre physis y nomos es, entonces, la condición de posibilidad de la distorsión y, por ello, de la política. Solo una concepción de physis como

${ }^{11}$ Ibídem 151.

${ }^{12}$ Rancière identifica, en sintonía con lo antedicho, que el régimen de lo Uno en tanto mutila lo múltiple proliferante conlleva siempre un odio a lo Otro. En sus palabras: "no es el consenso sino la exclusión; no es la razón devenida racionalidad de la coexistencia de las satisfacciones sino el puro odio de lo Otro, el reunir para excluir" Rancière, Jacques. "La fin de la politique ou l' utopie réaliste". Aux bords du politique. Francia: Gallimard, 2012. 23-74. 54.

${ }^{13}$ Rancière, Jacques. El desacuerdo. Op. Cit. 87. 
multiplicidad proliferante puede aprehender la excedencia constante de todo orden. Esta multiplicidad proliferante se incardina en la igualdad natural de los hombres. La igualdad de cualquiera con cualquiera que es renuente a toda realización. Como fundamento vacío la igualdad resiste la subsunción a todo mecanismo repetitivo, de lo Uno, del Mismo. La physis como algo imposible de ser aprehendido conceptualmente ni reducido a un modo sensible es lo que subyace a la excedencia constante de ésta frente al nomos. Solo una naturaleza no reductible a la ley del Uno permite la multiplicidad proliferante. Solo una naturaleza que demuestre ser siempre algo más que aquello que la ley pretende como natural, permite la puesta en cuestión de la ley misma. La definición de la naturaleza como potencia múltiple proliferante evita, así, toda identificación de ésta con aquello que se muestra como natural, que no es más que artificial.

La separación de physis y nomos, bajo una concepción de la naturaleza como multiplicidad proliferante, es el fundamento de la desidentificación de todo orden y reparto sensible con el orden y reparto sensible por naturaleza. No obstante esto, de la separación entre physis y nomos no se deriva un modo único de tramitar dicha distancia. Dos son los modos que Rancière restituye de tratar dicha distancia, es decir, de constatar que lo existente de hecho no se ajusta al derecho ${ }^{14}$. Un primer modo subordinado a lo que el autor identifica como silogismo de la sospecha y otro segundo referido como silogismo de la emancipación. Si ambos parten de la separación entre el hecho y el derecho -de la constatación que el orden existente no coincide con el orden natural- el razonamiento y la praxis que conllevan son diferentes:

\footnotetext{
${ }^{14}$ Rancière hace oscilar al concepto de derecho entre su versión positivista, derecho como ley escrita, y su concepción naturalista, derecho como lo justo por naturaleza. También traza una continuidad entre lo justo por naturaleza y una partición dada concebida como natural. Su despreocupación en torno a estabilizar el concepto de derecho y de physis nos permite mantener la misma actitud. Así, por ejemplo, si el consenso y el orden policial se caracterizaban por suturar el espacio entre physis y nomos, lo mismo hace entre derecho y hecho: "También son extensiones de la capacidad del Estado experto para poner a la política como ausencia suprimiendo todo intervalo entre derecho y hecho [...] la extensión de este proceso es la identificación creciente de lo real y lo racional, lo jurídico y lo erudito [...] que son en primer lugar las garantías del poder estatal, la seguridad siempre fortalecida de su infalibilidad, de la imposibilidad de que sea injusto [...] allí el derecho y el hecho se hacen tan indiscernibles como la realidad y su imagen, como lo real y lo posible" Ibídem. 141-142.
} 
El silogismo es, entonces, simple: en la mayor, aparece aquello que dice la ley; en la menor aquello que se dice o se hace en otro lugar, un hecho o una frase que contradice la afirmación jurídico-política fundamental de la igualdad. Pero existen dos maneras de pensar la contradicción entre la mayor y la menor ${ }^{15}$

El silogismo de la sospecha es vinculado por Rancière con la crítica de las ciencias sociales y, principalmente, su potencia desmitificadora. Si el texto jurídico proclama la igualdad de todos los hombres, si la comunidad se presenta como la realización del bien natural, el primer paso que hace el pensamiento de la sospecha es la señalar la distancia entre la proclamación y lo existente. Entre lo abstracto y lo concreto, entre la apariencia y la realidad. El silogismo de la sospecha "consiste en concluir simplemente que la frase jurídico-política es una ilusión, que la igualdad postulada es una apariencia que no es otra que la de enmascarar la realidad de la desigualdad" ${ }^{16}$.

Al modo de tramitar la distancia entre physis y nomos del pensamiento de la sospecha Rancière lo identifica con un nihilismo crítico cuya productividad se acaba allí dónde verifica la desigualdad: "pretendiendo explicitar la desigualdad, se la ha vuelto más rígida" ${ }^{17}$. El silogismo de la sospecha, entonces, denuncia el carácter aparencial de todo pueblo, de la proclamación de la igualdad y de la comunidad existente. Demuestra que en lo concreto la desigualdad es persistente, y por ello, la verifica. Que aquel orden proclamado natural no es más que un artificio. Pero este reconocimiento deviene impotente ya que solo está capacitado para señalar lo ilusorio, pero no de indicar el camino a seguir.

Frente a la impotencia de la sospecha Rancière opone la potencia de la emancipación. Si el silogismo de la sospecha denuncia el carácter ilusorio de la proclamación de la igualdad, del orden natural, reproduciendo, así, la desigualdad, el silogismo de la emancipación opera de modo distinto: verificando la igualdad. Esto implica

\footnotetext{
${ }^{15}$ Rancière, Jacques. "Les usages de la démocratie". Aux bords du politique. Op. Cit. 74-112. 87.

${ }^{16}$ Ibídem.

${ }^{17}$ Ibídem. 99.
} 
que la apariencia antes denunciada sirve ahora de fundamento para la irrupción de la parte sin parte en la comunidad. La proclamación de la igualdad de los humanos por naturaleza no es comparada con la realidad y denunciada como falsa. El procedimiento es el inverso: el hecho es comparado con la naturaleza -la igualdad de cualquiera con cualquiera- $y$, tras constatar su distancia -el fenómeno de la desigualdad- se pone en cuestión el orden en nombre de la naturaleza.

La distancia entre physis y nomos, entre derecho y hecho es la que le permite -en nombre de la igualdad natural de cualquiera con cualquiera, en nombre de la proclamación de la ley positiva de la igualdad de los hombres- poner en cuestión lo existente en tanto no coincide con lo proclamado. En este sentido "la proclamación igualitaria no es simplemente una pura nada" -una mera apariencia. Por el contrario, conlleva "el poder de crear un lugar en el que la igualdad pueda reclamarse de ella misma". Esto implica que la crítica no viene a demostrar el carácter aparencial de la igualdad. No denuncia la apariencia sino que la utiliza para irrumpir y verificarla: "aquello que debe ser demostrado, es precisamente la igualdad" ${ }^{18}$. No implica esto, claro está, que la distancia entre lo abstracto y lo concreto desaparezca. Esta distancia es la que permite la verificación continua de la igualdad. El derecho solo se realiza en la verificación fugaz de la igualdad.

En la apariencia hay una verdad que está negada y en tanto está como negada es la condición de posibilidad para su verificación: "le inventan un nuevo lugar: el espacio polémico de una demostración que reúne la igualdad y su ausencia” ${ }^{19}$. La irrupción de la parte sin parte ocurre allí donde el hecho demuestra no agotar lo natural y lo hace en nombre de la physis. Se funda en la igualdad natural pero no para realizarla de modo completo, solo para verificarla. Esta verificación se produce de modo conflictivo, polémico.

\footnotetext{
${ }^{18}$ Ibídem. 88.

${ }^{19}$ Rancière, Jacques. El desacuerdo. Op. Cit. 116.
} 
La denuncia de la impotencia de la sospecha, de que todo es apariencia, olvida que la igualdad natural es aquello que impulsa la acción política. No obstante esto, si hay una apelación a lo natural o a la idea de lo justo, es solo al modo de la irrupción polémica tal como es descripta por el silogismo de la emancipación. El derecho, la justicia, el pueblo no se fijan en instituciones, no se realizan más que en su demostración conflictiva de no ser aquello a lo que son reducidos. Se dan allí donde irrumpe una nueva partición de lo sensible: "La demostración del derecho o manifestación de lo justo es una nueva representación de la partición de lo sensible ${ }^{20}$.

Hasta aquí, hemos restituido cómo Rancière nos presenta tres modos de visualizar la relación entre physis y nomos: a) una primera que los identifica clausurando la posibilidad de la política misma; b) una segunda que, incardinada en el espacio entre ambos términos, denuncia el carácter aparencial de lo fáctico; y c) una tercera forma también emplazada en el espacio entre derecho y hecho que no se limita a verificar la apariencia de lo fáctico sino que fuerza a lo fáctico a verificar lo aparece. Si el segundo modo conlleva a un nihilismo desmitificador, el tercer modo, el de la emancipación, conlleva a la praxis política como modo de subjetivación de un actor político contingente, el pueblo, que irrumpe en la comunidad y muestra su carácter partido.

B) y c) Ttenen en común la desidentificación entre physis y nomos, y por eso permiten la crítica (impotente en el caso b) y que, en el c) tiende a la acción emancipadora)

A) y c) tienen común la apelación al concepto de physis. El orden policial o el consenso, apela a la physis para fundar y legitimar aquello que existe. La política, la emancipación, apela a la physis para irrumpir y poner en cuestión un reparto sensible dado. Pero esto solo puede hacerlo, en la medida que la physis excede todo orden y escapa a toda delimitación posible. En a) la physis es aprehensible y realizable completamente en el nomos, en c) physis y nomos, si se relacionan, es solo con el signo de

${ }^{20}$ Ibídem. 75. 
la negatividad, de la physis negando el nomos dado. Entre physis y nomos hay una dicotomía que es la condición de posibilidad de la política misma.

Es necesario ver en qué medida la propuesta de un concepto de naturaleza como potencia múltiple proliferante se asienta en los pilares del excepcionalismo y la identificación entre política y conflicto. En palabras de Rancière: “Para que haya política se necesita un título de excepción, un título que se agregue a aquellos por los cuales se rigen 'normalmente' las sociedades". Esta "excepción ordinaria [es] el poder del pueblo" ${ }^{21}$ y su manifestación es la irrupción conflictiva. Es decir, la política es una "situación extrema" que "escapa a la medida ordinaria" donde el pueblo "se declara como excepción radical a la comunidad", como "parte excepcional" irrumpiendo de modo conflictivo e interrumpiendo la distribución de las partes existente ${ }^{22}$. Esto implica que si la manifestación del pueblo es un hecho comunicacional en tanto toma de palabra, a su vez "escapa a las distinciones que fundan las reglas supuestamente 'normales' de la discusión" ${ }^{23}$.

El excepcionalismo es un corolario de la comprensión específica del modo en que physis y nomos se realizan. Si la physis solo se realiza en la verificación fugaz de la igualdad, si siempre debe permanecer como excedente de todo orden para poder ponerlo en cuestión, la visibilidad de ésta corresponde, temporalmente, a lo excepcional. La fugacidad es idéntica a su excepcionalidad. Y esa fugacidad se manifiesta en la irrupción del conflicto. Mientras que el tiempo de lo normal se manifiesta en el consenso.

Dicho esto, la viabilidad de superar la oposición normalidad-excepción en Rancière se subordina a la posibilidad de ir más allá de la dicotomía policía-política, nomos y physis. A la posibilidad de reconocer que las variaciones de signo de los regímenes policiales son significativos en la medida que, el orden policial, es el único modo que alberga una temporalidad más allá de la fugacidad excepcional. Es el propio Rancière quien identifica el peligro de un pensamiento dicotómico tal y esboza una salida. En sus palabras, si bien:

\footnotetext{
${ }^{21}$ Rancière, Jacques. El odio a la democracia. Buenos Aires: Amorrortu, 2006, p. 74.

${ }^{22}$ Rancière, Jacques. El desacuerdo. Op. Cit. 10, 30, 33, 56.

${ }^{23}$ Ibídem. 76.
} 
la práctica de los escitas que vaciaban los ojos de sus esclavos y la de las modernas estrategias de información y la comunicación [...] competen ambas a la policía[,] no se sacará de ello en modo alguno la conclusión nihilista de que una y otra son equivalentes. Nuestra situación es en todos los aspectos preferibles a la de los esclavos escitas $^{24}$

Con esto en mente, la oposición entre policía y política puede ser comprendida: a) de un modo nihilista en cuanto esté incapacitado de observar las diferencias entre regímenes policiales ${ }^{25}$; b) superando el nihilismo en tanto examinar tales diferencias.

Es de suponer, en la medida que es quien introduce los criterios y marca los peligros de la posición a), que Rancière cabe en la segunda posibilidad. En sintonía con ello enuncia que "hay una policía menos buena y una mejor" y nos presenta el criterio de juicio, donde el concepto de physis aparece:

la mejor no es, por lo demás, la que siguen el orden supuestamente natural de las sociedades o la ciencia de los legisladores sino aquella a la que las fracturas de la lógica igualitaria llegaron a apartar las más de las veces de su lógica "natural”26.

La mejor policía es aquella que se asienta en el hecho de no apoyarse en un orden supuestamente natural sino que deja albergar, en sí, las fracturas de la lógica igualitaria. Es decir alejarse de la lógica "natural" de la policía que es identitaria. Una policía mejor que otra es una policía menos policial, aquella que permite el juego de la irrupción en sí mismo. Esto implicaría, dejando de lado las dificultades de instituir un juego de irrupciones, la contaminación entre la policía y la política.

${ }^{24}$ Ibídem. 46.

${ }^{25}$ Lo que implica un segundo modo del nihilismo en la obra de Rancière. Uno primero asignado al silogismo de la sospecha que denunciando del carácter aparencial de lo existente, no hace más que reproducir la injusticia. Un segundo modo, entonces, que apunta a la imposibilidad de distinguir las variaciones entre órdenes policiales.

${ }^{26}$ Ibídem. En el original "Il y a de la moins bonne et de la meilleure police - la meilleure, au demeurant, n'etant pas celle qui suit l'ordre suppose naturel des societes ou la science des legislateurs mais celle que les effractions de la logique egalitaire sont venues le plus souvent ecarter de sa logique 'naturelle' La police peut procurer toutes sortes de biens, et une police peut etre infiniment preferable a une autre. Cela ne change pas sa nature qui seule est ici en question" 
El mismo Rancière, en la oración siguiente, da un paso atrás: “Una policía puede ser infinitamente preferible a otra. Esto no cambia su naturaleza, que es lo único está en cuestión aquí" 27 . Paso atrás que implican dos corolarios: 1) Si su naturaleza es invariable, ¿cómo "apartarse las más de las veces de su lógica "natural"? ¿cómo pensar los intersticios entre ambas? Si la naturaleza de los órdenes es invariable, se torna imposible toda contaminación, se torna imposible el alejamiento antes mencionado; 2) Si la naturaleza invariable de los órdenes es lo único [le seule] que importa analizar en el texto, la viabilidad de superar la dicotomía policía-política, ordinario-extraordinario, normalidadexcepción, no es sujeto de interés en el trabajo o, lo que en sus consecuencias es idéntico, ocupa una parte ínfima en la economía del texto. Ocupación ínfima que repercute en el modo de recepción del texto, es decir, en el reforzamiento de la hermenéutica dicotómica y en la imposibilidad de superar el excepcionalismo y la identificación entre política y conflicto.

Si, según Rancière, la oposición entre policía y política es posible de comprenderse de dos modos -uno nihilista en cuanto desatento a las diferencias entre los regímenes policiales y otro, por lo pronto no nihilista, en tanto atento a aquellas variaciones- lo cierto es que su esfuerzo teórico torna no nos brinda los elementos para juzgar aquellas diferencias. Esto por su énfasis en el carácter invariable de la naturaleza de los órdenes y su mutua heterogeneidad. Para Rancière a pesar de que "la política se topa en todos lados con la policía[,] es preciso pensar este encuentro como encuentro de los heterogéneos" ya que es "una lógica completamente heterogénea a la de la policía". Lo que conlleva "renunciar al beneficio de ciertos conceptos que aseguran de antemano el pasaje entre los dos dominios" 28 . La renuncia a conceptos que rompan con la dicotomía y la acentuación del carácter completamente heterogéneo de los órdenes, hace imposible pensar lo que Rancière había propuesto como criterio de juicio: "que las fracturas de la lógica igualitaria llegaron a apartar las más de las veces de su lógica 'natural'”29. Por ello, si Rancière evita caer en las derivas nihilistas de la dicotomía al poder distinguir entre ordenes policiales

\footnotetext{
${ }^{27}$ Rancière, Jacques. El desacuerdo. Op. Cit. 46.

${ }^{28}$ Ibídem. 47.

${ }^{29}$ Ibídem. 46.
} 
mejores que otros, su teoría no lo permite. La persistencia de la dicotomía, el énfasis en la heterogeneidad y la imposibilidad de conceptualizar los intersticios, hace imposible escapar de las derivas nihilistas de su teoría, en principal, de su lectura y recepción.

Hasta aquí, vimos entonces:

1) Que Rancière nos presenta tres modos de visualizar la relación entre physis y nomos: a) un modo consensual que los identifica de modo total; b) un modo nihilista identificado con la crítica desmitificadora de la crítica de las ciencias que separa physis y nomos para demostrar la inexistencia de lo natural, de que todo es apariencia; y c) una tercera forma también emplazada en el espacio entre physis y nomos que no se limita a verificar la apariencia de lo fáctico sino que fuerza a lo fáctico a verificar la igualdad natural.

2) Que una concepción de la naturaleza como potencia múltiple proliferante, renuente a toda identificación con el nomos, habilita a concepción de la política cuya temporalidad es la de lo excepcional, que solo existe en el irrumpir y dura lo que éste dure. Que ésta temporalidad conlleva una intensidad alta, es decir, que se da al modo del conflicto. Y que una concepción tal parece derivar en una lectura dicotómica de policía y política. Es el propio Rancière el que llama la atención sobre los peligros de esta dicotomía, por lo que nos detuvimos en su intento de escapar de estos.

3) Que el intento de Rancière de escapar de los peligros nihilistas de la dicotomía entre política y policía no se traduce en una teoría que permite vislumbrar mejores y peores órdenes policiales. Que el único esbozo que Rancière realiza -donde afirma que el mejor orden policial es el que más permite la irrupción de la política o menos policial esluego se vuelve impensable dentro de su teoría en la medida que renuncia a pensar conceptos que se sitúen entre ambos modos, terminando por sostener que política y policía portan naturalezas heterogéneas que no pueden contaminarse. Por ello, sostuvimos que, si el silogismo de la sospecha lleva a un nihilismo desmitificador pero pasivo en tanto se limita a verificar la desigualdad, el tercer modo, el de la emancipación, 
encuentra como su corolario un nihilismo activo de la irrupción. Un nihilismo que, asentado en el excepcionalismo, incapaz de distinguir entre regímenes mejores que otros solo obtiene como correlato una política de la negatividad. De esta forma los modos de lo normal, de lo ordinario, se vuelven imposibles de ser pensados. Un nihilismo que prescribe: te opondrás a todos los regímenes ya que ninguno es natural.

La posibilidad de desarticular el excepcionalismo y la identificación entre política y conflicto es coetánea a la aceptación de la posibilidad de una política de lo positividad. Una política de lo positivo o de la positividad implica una política que supere la puesta en cuestión de los órdenes vigentes, que supere una política de develación del carácter parcial del orden y su puesta en cuestión. No supone su anulación, pero si su posición privilegiada. Es que tras el momento de negación, la positividad se impone. En tanto la negatividad no se puede institucionalizar, o en los términos de Rancière, "la persistencia de ésta [la distorsión] es infinita porque la verificación de la igualdad es infinita” ${ }^{30}$, una política de la negatividad le concede a la política de signo contrario la arena de construcción de lo ordinario, de lo normal, de lo positivo. Como corolario se logra una teoría y filosofía política corta de miras, incapaz de visualizar variaciones significativas.

Pensar una política de lo ordinario implica retornar a la posibilidad de una identificación entre physis y nomos. El primer peligro que se nos aparece, o que en todo caso no nos dejaran olvidar los partidarios de la negatividad, es la igualación entre ambos polos. Es que en tanto que la naturaleza siempre excede a su manifestación sensible su identificación implica su reducción. El peligro de identificar institución y justicia, es su parcialidad, su finitud. Pero ese peligro es diferenciable de la equiparación total entre la justicia existente y la justicia posible 0 , en los términos de Rancière, la suposición del cierre del campo comunitario a través de la representación del pueblo. Ahora bien, podríamos preguntarnos si toda realización de justicia implica la anulación del espacio entre physis y nomos. En otras palabras, si toda positividad implica la igualación entre lo positivo y lo natural, lo fáctico y lo válido. Si el interrogante es respondido de modo

\footnotetext{
${ }^{30}$ Rancière, Jacques. El desacuerdo. Op. Cit. 57.
} 
negativo, otro aparece: si el espacio entre physis y nomos no es anulado, ¿es posible anudarlo de un modo que no sea el de la irrupción? ¿Puede anudarse sin llegar a ser una identidad plena? Es en este sentido, para responder estos interrogantes, que Leo Strauss aparece en escena.

La legitimidad de la utilización de Leo Strauss viene dada, como dijimos, por su introducción vía Rancière. Es éste quien polemiza de modo directo con Strauss identificándolo con los intentos de un retorno de la filosofía política y, por ende, de la arquipolítica platónica. Es que su proyecto implica la realización de un "principio eterno de una política del bien común y de la educación ciudadana que Leo Strauss opone al hundimiento utilitarista moderno"31. Implica, entonces, la realización del arkhe comunitario bajo la restitución de lo justo y lo injusto por naturaleza, es decir, el derecho natural. Si Leo Strauss es identificado con la restitución de la pregunta por lo justo y lo injusto bajo la indagación racional del derecho natural de la filosofía política, el desacuerdo es referido como la polémica y la irrupción de la parte reducida a mera phoné que demuestra el carácter no natural da la partición. Con todo esto, podemos decir que si el intento straussiano es el de identificar physis y nomos mediante la interrogación y deducción racional de lo natural, la propuesta de Rancière implica la puesta en cuestión de la deducción pretendidamente racional del orden justo por naturaleza. Una vez más, de la equiparación entre physis y nomos.

En lo que sigue se pretenderá complejizar la lectura rancieriana de Leo Strauss. No por ímpetu de corrección de lectura. En tanto Rancière no es un comentarista de Strauss la rectitud de su lectura per se no representa problema alguno. Ahora bien la simplificación del argumento straussiano, bajo la identificación entre physis y nomos, obstruye un modo de pensar la relación entre ambos términos que escapa a los tres que se encuentran en la obra de Rancière. Dicho de otro modo, la problematización de la lectura que Rancière hace de Leo Strauss nos permite pensar un modo de relacionar

${ }^{31}$ Ibídem. 14. 
physis y nomos que al mismo tiempo habilite una separación entre ellos sin renunciar a su anudación.

\section{Las enseñanzas de la filosofía política}

La posibilidad de una política positiva, ligada a su institucionalización, que no caiga en la igualación entre la justicia realizada y la posible, necesita de una articulación entre physis y nomos no trabajada por Jacques Rancière. Necesita, entonces, de un modo que los anude sin igualarlos. Una identificación sin igualación real. Es en este sentido que restituiremos el argumento de Leo Strauss presentado en la conferencia “¿Qué podemos aprender de la teoría política?" pronunciada en 1942 en la New School de Nueva York. El mismo Leo Strauss se encarga de rectificar el título y reemplazarlo por “¿Qué podemos aprender de la filosofía política?". Veamos entonces, que podemos aprender de la filosofía política.

Leo Strauss comienza la conferencia enunciando tres argumentos por los cuáles la filosofía política no puede enseñarle nada a la práctica política. A saber: "1)Cabe dudar que exista tal cosa que merezca ser llamada filosofía política; 2) aun cuando existiera una filosofía política, no la necesitaríamos; 3) aun cuando la necesitásemos sus lecciones carecerían inevitablemente de eficacia” ${ }^{32}$. De modo paradójico el mismo Strauss admite la plausibilidad de todos los argumentos. Frente a la falta de univocidad de la respuesta desde la filosofía política al problema político afirma que ésta es "en el mejor de los casos, la posesión de un conocimiento claro acerca de los problemas, no la posesión de un conocimiento claro acerca de las soluciones de los problemas". En otras palabras "las cuestiones verdaderamente filosóficas, permanecen sin respuesta" ${ }^{33}$. Frente al carácter no necesario de la filosofía política afirma "no tengo la menor duda respecto a la posibilidad de planificar una política internacional inteligente sin necesidad de recurrir a la filosofía política" ${ }^{34}$. Existe una diferencia clara entre filosofía política y sabiduría práctica.

${ }^{32}$ Strauss, Leo. “¿Qué podemos aprender de la teoría política? ¿Qué es filosofía política? y otros ensayos. Madrid: Alianza, 2014. 154-183. 157.

${ }_{33}^{33}$ Ibídem. 158.

${ }^{34}$ Ibídem. 160. 
Por último, frente a la tesis de la ausencia de eficacia de la filosofía política para los problemas políticos afirma: "la filosofía siempre llega demasiado tarde para guiar la acción política [...] no puede hacer más que interpretar el resultado de la acción política”35.

El interrogante por la posibilidad de aprender algo de la filosofía política parecería tener, así, una respuesta negativa. No obstante esto, durante la conferencia Strauss tuerce el rumbo de la misma. Es una de las enseñanzas restituidas por el autor la que reviste de mayor importancia para nuestro problema. Es aquella que gira en torno al descubrimiento filosófico de la naturaleza, del derecho natural. Pero comprender cabalmente el derecho natural implica comprender su contrario, "de qué se diferencia y a qué se opone dicho término". La respuesta, claro, "la convención o la ley"36. El descubrimiento de la naturaleza implica reconocer su oposición a lo artificial o convencional. Tras esto, al notificar el carácter idéntico del ardor del fuego en todas las ciudades, en tanto anclado en la naturaleza, y el carácter diverso de las leyes de las mismas ciudades donde el fuego arde, la filosofía política se interrogó por la posibilidad de encontrar en la naturaleza, un "orden de vida que fuese bueno y justo en todas partes [...] un orden natural [que fuera] el único criterio verdaderamente legítimo para juzgar" 37 .

La interrogación por el derecho natural implica la destrucción de la identidad entre lo bueno y lo heredado, la reducción de lo existente a convención. Si hasta entonces la tradición fungía como parámetro de lo justo, la primera lección de la filosofía política se apoya en su fuerza antitradicional. En tanto interrogación del orden por naturaleza la filosofía "presupone, por ende, la duda respecto de la autoridad" ${ }^{38}$. Esto implica que los filósofos "prevenían a quienes querían escucharles de identificar cualquier orden existente, por más satisfactorio que fuese en muchos aspectos, con el orden perfecto" ${ }^{39}$.

\footnotetext{
35 Ibídem. 161.

${ }^{36}$ Ibídem. 166.

${ }^{37}$ Ibídem.

${ }^{38}$ Strauss, Leo. Derecho natural e historia. Buenos Aires: Prometeo, 2014. 136.

39 Strauss, Leo. “QQué podemos aprender de la teoría política?” Op. Cit. 167. O, como dice en el "Prefacio a Crítica de la religión de Spinoza": "Se pueden resolver los problemas finitos y relativos; los problemas absolutos no pueden resolverse. En otras palabras, los seres humanos nunca crearán una sociedad libre de contradicciones. Strauss, Leo "Prefacio a Crítica de la religión de Spinoza" En Liberalismo antigui y moderno. Katz: Buenos Aires, 2007. 321-371. 330.
} 
La interrogación por el orden natural devela el carácter netamente revolucionario, o cuánto menos, crítico de la filosofía política.

Con esto dicho, la primera lección de la filosofía política posee una forma análoga al silogismo de la emancipación en Rancière. La apelación a lo natural tiene el sentido de develar limitado, y por tanto criticable, lo convencional. La primera lección de la filosofía política nos advierte del carácter excedente de la physis respecto del nomos. Así, lejos de suturar el espacio entre ambos términos la postura straussiana parecería conducir a la irrupción rancieriana. La lectura que Rancière hace de Strauss sería "corregida" a costa de olvidar la posibilidad una articulación diferente a las propuestas por el francés entre physis y nomos.

Ahora bien, ésta no es la última palabra de la filosofía política al respecto. El carácter antitradicional de la filosofía política clásica es acompañado por una prudencia. Lo que implica que si bien "nunca ha habido ni habrá una época en que la medicina administrada por la filosofía política haya sido o será superflua" -es decir que la physis pueda ser realizada íntegramente en el nomos- "debe siempre administrarse, como toda medicina, con prudencia” ${ }^{40}$. Es necesario analizar de modo detallado en qué consiste la prudencia filosófica que parecería llevarnos a la enseñanza de la misma.

Nos dice Strauss que "los filósofos a los que me acabo de referir denominan al orden perfecto de la sociedad como un objeto eukhé, que significa tanto deseo como ruego". Esto implica que si bien "no dejaron lugar a dudas sobre el abismo que separa el ideal de la realidad" -una vez más, la distancia entre pyhsis y nomos entre derecho y hecho- "no existe ninguna razón necesaria por la cual deba hacerse realidad"41. Si el primer impulso de la constatación de la distancia entre la convención y la naturaleza nos llevaba a la fuerza antitradicional de la primera, el segundo impulso nos llama a la prudencia. Es que la imposibilidad de fundar el orden perfecto, de hacer el cielo en la tierra, nos recuerda el hecho de que es en la imperfección únicamente dónde transitamos

\footnotetext{
${ }^{40}$ Strauss, Leo. “QQué podemos aprender de la teoría política?” Op. Cit. 167.

${ }^{41}$ Ibídem. 168.
} 
la vida. Aun a sabiendas de que el orden natural, la physis, excede a todo orden convencional, la apelación al primero para poner en cuestión el segundo puede pecar de imprudente.

Con esto, parece vislumbrarse otro modo de relación entre physis y nomos que escape a los tres restituidos por Jacques Rancière, a saber: a) La fusión íntegra entre ambos términos que implica la arquipolítica platónica y todo régimen consensual; b) la develación de lo existente como aparente emparentada con el silogismo de la sospecha; c) la puesta en cuestión de lo existente mediante la verificación de la igualdad.

Es en la filosofía política clásica donde encontramos un cuarto modo de relacionar ambos términos. Es necesario citar in extenso:

La relación del ideal -o de la utopía- con la realidad, tal como ellos la concebían, podría ser descrita de este modo: existe una justicia civil común y ordinaria que consiste en obedecer la ley del país y en administrar de forma justa esa ley; esa justicia no se preocupa por la justicia de la propia ley; por esa razón se trata de una justicia muy imperfecta, ya que toda ley o todo ordenamiento jurídico está destinado a ser sólo de forma imperfecta justo; por consiguiente, la justicia debe ser complementada con la equidad en tanto que corrección de la justicia legal orientada a la justicia perfecta [...] la equidad o caridad no son capaces, por sí mismas, de subsistir en este mundo sin la sólida, algo brutal e imperfectamente justa infraestructura de una justicia común; la justicia común debe ser "completada" y corregida por razones de equidad o caridad, no puede nunca ser suplantada por ellas $^{42}$.

La lección de la filosofía política, de este modo, apunta a la prudencia. Prudencia cuyo punto nodal implica el reconocimiento que aunque la justicia del mundo es imperfecta, representa la única apoyatura para una justicia posible. Physis y nomos de este modo se anudan sin igualarse. Una relación dinámica, aunque prudente, se abre de este modo. Esto implica que la apelación a la physis excedente no apunta a la verificación

${ }^{42}$ Ibídem. 169. 
de la parte sin parte que cristaliza el litigio en la comunidad si no, más bien, a modos intermedios de la misma. El movimiento entre lo natural y lo convencional, entre el orden perfecto y sus realizaciones imperfectas no implica apelar al primero para destruir el segundo. Pero tampoco implica la plena realización del physis en el nomos como pretende Rancière.

Es esta idea, de lo intermedio, a la que apunta Leo Strauss en Derecho natural e historia, principalmente en su capítulo dedicado al derecho natural clásico. Allí sostiene que "para evitar malentendidos, es necesario decir algunas palabras sobre la respuesta característica de los clásicos a la pregunta por el mejor régimen" ${ }^{43}$. En tanto “el mejor régimen es solo posible bajo las condiciones más favorables [...] solo son posibles y, por ende, legítimos, regímenes más o menos imperfectos". Esto implica que "los regímenes legítimos o justos son posibles y moralmente necesarios en todo tiempo y lugar" ${ }^{44}$. Tras la constatación de la distancia entre el régimen del filósofo y el de toda ciudad existente, pero a su vez que solo las ciudades existentes son la condición de posibilidad de cualquier filosofía, el filósofo se vuelve prudente. Es que "el filósofo admite que lo más elevado intrínsecamente o por naturaleza no es lo más urgente para el hombre, que es en esencia un ser 'intermedio' -entre las bestias y los dioses" ${ }^{45}$. El carácter intermedio del hombre exige "diluir el derecho natural en el derecho meramente convencional" ya que, de otro modo "el derecho natural operaría como dinamita para la sociedad civil" 46.

En tanto ser un entre, toda producción de justicia, todo intento de desarrollar una ciudad justa, debe aceptar su carácter finito. Debe aceptar que "lo políticamente bueno es aquello que 'elimina una enorme cantidad de mal sin conmover una enorme cantidad de prejuicios $^{\prime \prime \prime 7}$. Si el primer impulso de la filosofía política apunta a destruir la tradición, prudencia mediante, necesita aliarse a ella. Es que la posibilidad de una justicia en el mundo necesitará de una mediación entre lo existente y lo ideal, por ello "algo

\footnotetext{
${ }^{43}$ Strauss, Leo. Derecho natural e historia. Op. Cit. 185.

${ }^{44}$ Ibídem. 184.

${ }^{45}$ Ibídem. 195.

${ }^{46}$ Ibídem. 195-196.

${ }^{47}$ Ibídem. 196.
} 
políticamente bueno, es por así decirlo el cociente entre lo que es simplemente bueno y lo ancestral” ${ }^{48}$, entre el derecho y el hecho, entre physis y nomos. El dinamismo entre orden y conflicto parece, así, adquirir matices. La posibilidad de una apelación a la relación entre physis y nomos que no renuncie a su articulación y que, a la vez, sepa de su imposible igualación, nos permite pensar un modo prudente de la acción política. La escala de tonalidades entre el consenso apolíneo y la política dionisiaca se vuelve pensable de este modo.

\section{Conclusiones}

El presente trabajo nació de la motivación de ir más allá dos pilares del pensamiento político posfundacional en particular y, más precisamente, de su retórica y del lenguaje del pensamiento político en general. Interrogación incardinada en la aseveración de una distancia entre la retórica posfundacional y su teoría. Si el posfundacionalismo reconoce tanto la necesidad como la imposibilidad de los fundamentos, la pregunta por la variación de los fundamentos, los signos de los mismos, ha sido relegada al olvido. Si la existencia humana es un continuo movimiento entre un orden y su puesta en cuestión, positividad y negatividad, es el momento de la apertura a algo otro el que ha hegemonizado la economía textual y discursiva del pensamiento político contemporáneo. Así, determinados conceptos han ganado una valoración positiva en detrimento de otros. La acentuación del momento negativo ha significado el olvido del momento ordinario. En términos temporales, si la negatividad porta el tiempo de la excepción, de lo extraordinario y la positividad el de la norma y de lo ordinario, la teoría y filosofía política contemporánea ha dejado en segundo plano el tiempo por excelencia de la vida. O el tiempo donde más transcurre la vida. La acentuación en el excepcionalismo y la equiparación entre política y conflicto ha obliterado pensar los modos de lo normal, las relaciones no armoniosas entre politizaciones y despolitizaciones constantes ${ }^{49}$.

${ }^{48}$ Ibídem.

49 Aquí es nuestro lenguaje conceptual el que nos tiende una trampa y realiza una conversión ilegítima entre cuantitativo y cualitativo. Politizar y despolitizar hace a un continuo de intensidad que iría del conflicto al consenso. En el continuum despolitizado-politizado mientras más nos acercamos al extremo 
Para ello restituimos los modos en los cuales Rancière presenta las posibles relaciones entre physis y nomos con sus respectivas derivaciones político-prácticas. El modelo del consenso, del régimen de lo Uno identifica plenamente physis y nomos suturando así el espacio comunitario y negando, en los términos de Rancière, la posibilidad de la política, es decir, del desacuerdo. Frente a esta presunta realización íntegra de la physis y nomos Rancière opone el silogismo de la sospecha y el silogismo de la emancipación. El primero, denuncia el carácter aparencial y artificial de aquello que aparece como natural, pero al ser este su único movimiento no hace más que reproducir la desigualdad. Por ello la potencia crítica desmitificadora deriva en un nihilismo pasivo que se limita a verificar la injusticia existente. El silogismo de la emancipación se ubica, al igual que el de la sospecha, en la distancia entre physis y nomos pero utiliza la contradicción entre ambos para la potencia de la irrupción de la parte de los sin parte en el seno de la comunidad. Si de la crítica desmitificadora se deriva un nihilismo pasivo, la irrupción emancipadora tiene como correlato un nihilismo activo. Nihilismo activo representado por la incapacidad de distinguir teóricamente las variaciones de los órdenes policiales a pesar de la afirmación de Rancière.

Los modos de relacionar physis y nomos que nos presenta Rancière hace imposible pensar una política de la positividad que no evite, a su vez, el cierre total del campo comunitario. A su vez que la afirmación de la heterogeneidad completa entre el orden policial y la política vuelve imposible analizar los entres que hace al funcionamiento normal de la vida lo que lo lleva a un nihilismo teórico aunque activo. Por ello, nos preguntamos por la condición de posibilidad de una política de la positividad. Más en específico, nos interrogamos por la posibilidad de una relación entre physis y nomos que permitiera identificarlos sin clausurar su distancia. Ello nos llevó al modo en el cual Leo Strauss comprende la relación entre naturaleza y artificio. Modo que, aun a sabiendas del carácter excedente de la naturaleza respecto a su realización sensible no renuncia a su

politizado más elementos de "política" parecería haber. Pero a su vez despolitizar es una decisión política, conlleva un reverso político. En esta afirmación, que despolitizar es una decisión política, lo político deja de conceptualizar un quantum de intensidad. Implicaría una reflexión más acabada, pero cuanto menos, la política es un cruce entre intensidades plurales (politizaciones de aspectos y despolitizaciones de otros) motivadas por algo otro que no es medible en intensidades. 
articulación. $\mathrm{O}$, en otras palabras, que las identifica sin igualarlas plenamente. Lo que permite, al mismo tiempo, una relación dinámica entre physis y nomos sin que dicha relación sea solo de la índole de la irrupción. Este modo de relación se incardina en la prudencia de la filosofía clásica, prudencia que a sabiendas del carácter intermedio del hombre reconoce que la única justicia posible es la justicia en la tierra. Que toda justicia necesita apoyarse en órdenes imperfectos. Por ello si el primer impulso de la filosofía política es cuestionar la tradición, el segundo, prudencia mediante, es interrogar los modos de anudar tradición y derecho natural. Lo que Leo Strauss denomina lo políticamente bueno.

Recordemos los tres argumentos en torno a la inutilidad de la filosofía política para la práctica política: 1) La filosofía política no nos brinda un contenido positivo, no existe en singular: la filosofía política no existe; 2) Aun existiendo sería irrelevante, la práctica política se apoya en otros saberes; 3) Aun si existiera y fuera relevante, siempre vendría $a$ posteriori de la práctica política. Recordemos también que el propio Strauss acepta en parte la veracidad de las críticas, no obstante esto, no renuncia a la plausibilidad pedagógica de la filosofía política. Dicha enseñanza se asienta en la prudencia, en la mostración de “cuán extraordinariamente difícil es asegurar esos mínimos de decencia, de humanidad y de justicia que han sido dados por sentado, y siguen dándose por sentados $^{\prime 50}$. Dicha enseñanza, entonces, nos recuerda la irresponsabilidad de una política de la mera negatividad, de la puesta en cuestión sin observar a qué es lo que se opone. No significa un conformismo pasivo ya que "al moderar así nuestras esperanzas, nos protege contra el desaliento". Pero a la vez, "al inmunizarnos, así, contra la arrogancia de los filisteos, nos hace al mismo tiempo inmunes a los sueños del visionario" ${ }^{51}$. La filosofía política, entonces, nos recuerda el carácter siempre imperfecto de lo existente al mismo tiempo que sienta las bases para la interrogación de las variaciones de aquella imperfección. La prudencia, la constatación de que lo concreto no se ajusta a lo abstracto, de que toda justicia encuentra como apoyatura el mundo precario, es la condición de

\footnotetext{
${ }^{50}$ Strauss, Leo. “¿Qué podemos aprender de la teoría política?” Op. Cit. 179.

${ }^{51}$ Ibídem.
} 
posibilidad de una política de la positividad. De un pensamiento político que no renuncie a pensar el orden y sus modos. A su vez que, al moderar nuestras esperanzas, nos permite una actitud de mayor gratitud frente a los logros que supimos alguna vez conseguir, sin que esto implique una detención de la política.

\section{Bibliografía}

- Marchart, Oliver (2009). El pensamiento político posfundacional. Buenos Aires: FCE.

- Nosetto, Luciano (2015). “Jacques Rancière, Leo Strauss y el sentido del idealismo platónico", Leviathan | Cuadernos de Investigación Política, N. 10, pp 59-79.

- Rancière, Jacques (2006). El odio a la democracia. Buenos Aires: Amorrortu,

- Rancière, Jacques (2012). El desacuerdo. Política y filosofía. Buenos Aires: Nueva Visión.

- Rancière, Jacques. (2012) “La fin de la politique ou 1' utopie réaliste". Aux bords du politique. Francia: Gallimard, pp. 23-74.

- Rancière, Jacques (2012). "Les usages de la démocratie". Aux bords du politique. Francia: Gallimard, pp. 74-112

- Strauss, Leo (2007). "Prefacio a Crítica de la religión de Spinoza", en Liberalismo antiguo y moderno. Katz: Buenos Aires, pp. 321-371

- Strauss, Leo (2014). Derecho natural e historia. Buenos Aires: Prometeo.

- Strauss, Leo (2014). “¿Qué podemos aprender de la teoría política?, en ¿Qué es filosofía política? y otros ensayos. Madrid: Alianza, pp. 154-183. 